\title{
Risk factors for disc height loss in conservatively treated symptomatic lumbar disc herniation in elderly patients
}

\author{
Dong-Min Seo, Yongjae Cho \\ Department of Neurosurgery, Ewha Womans University College of Medicine, Seoul, Korea
}

Objective: The purpose of this study was to evaluate the clinical and radiologic factors associated with disc height loss in patients with spontaneous resolution of lumbar disc herniation.

Methods: In total, 56 symptomatic herniated lumbar disc patients above 65 years old (36 male and 20 female patients) who received conservative treatment from January 2017 to December 2018 were retrospectively investigated. Clinical findings including age, sex, pain, and smoking history were examined in each group (group A, patients with unchanged disc height; group B, patients with decreased disc height). As radiologic findings, the level, severity, laterality, Modic changes, and disc degeneration of lumbar disc herniation were investigated and compared between groups.

Results: Group A contained 30 patients and group B comprised 26 patients. No statistically significant differences were found in age, sex, visual analogue scale scores, level of disc herniation, and laterality of disc herniation between groups. Group A had more protrusion cases (14/30 cases) and group B had more sequestration (11/26 cases). Group B had more cases of Modic changes and more disc degeneration cases than group $A$.

Conclusion: In patients who receive conservative treatment for symptomatic lumbar disc herniation, disc height loss may occur later, especially in cases of extrusion- and sequestration-type herniation. This disc height decrease after lumbar disc herniation without surgical removal of the disc may occur due to disc degeneration and disc herniation itself. Patients who are treated conservatively should receive an explanation that disc height may be reduced later.

Keywords: Lumbar vertebrae; Intervertebral disc disease; Conservative treatment

\section{Introduction}

Lumbar disc herniation, which causes low back pain and radiating pain in the lower extremities, is a common disease that occurs in $20 \%$ to $30 \%$ of the population [1]. Since Mixter and Barr introduced surgical treatment for lumbar disc herniation in 1934, discectomy has been performed as standard treatment for this disease [2]. However, it has been reported that the lumbar disc herniation-induced low back pain and radiating pain in the lower extrem-

Received: July 7, 2021

Revised: October 8, 2021

Accepted: October 19, 2021

Corresponding Author: Yongjae Cho, MD, PhD

Department of Neurosurgery, Ewha Womans University Mokdong Hospital, 1071 Anyangcheon-ro, Yangcheon-gu, Seoul 07985, Korea

Tel: +82-2-2650-2685; Fax: +82-2-2650-2651;

E-mail:yongcho@ewha.ac.kr ities improves spontaneously without surgical treatment $[3,4]$. In 1984, Guinto et al. [4] reported for the first time that the herniated intervertebral disc disappeared spontaneously through computed tomography imaging [5-7]. Following this, numerous cases of spontaneous recovery have been published. This has been the basis for conducting conservative treatment for lumbar disc herniation $[4,8]$. Disc space height was decreased after discectomy due to surgical reduction of disc material, which have been widely known. But, few studies have assessed changes in disc height in patients who are treated conservatively $[5,9,10]$.

In this study, we retrospectively analyzed patients whose herniated lumbar disc disappeared spontaneously after conservative treatment and assessed the change in disc space height. The main purpose of this study was to evaluate the clinical and radiologic factors of disc height loss in spontaneous resolution of herniated lumbar disc patients. Demographic, clinical, and radiologic find- 
ings such as the level, status, severity, Modic change, and disc degeneration of lumbar disc herniation were assessed.

\section{Material and Method}

\section{Patients}

We retrospectively analyzed 56 symptomatic herniated lumbar disc patients from 226 patients above 65 years old who were diagnosed with acute symptomatic herniated lumbar disc from January 2017 to December 2018 in our department. Fifty-six patients (36 male and 20 female patients) were retrospectively chosen for this study. Patients selection criteria included: $(1)<50$ years of age with buttocks and leg pain concomitantly associated with a herniated lumbar disc; (2) one level of herniated lumbar disc (protrusion, extrusion, and sequestration) diagnosed on lumbar magnetic resonance imaging (MRI); (3) conservative treatment choice (medication and physical therapy) after medical explanation; and (4) regular hospital visits for 2 years with lumbar MRI during follow-up after initial MRI. The exclusion criteria were as follows (1) spine trauma history, (2) history of cancer, (3) previous lumbar surgery and (4) a change in treatment options, such as interventional block and surgery. Disc heights (initial and last follow-up MRI) were measured at T2 sagittal MR using modified method from Farfan (Fig. 1) [3,4,9]. Disc heights were defined as sum of the heights of anteroinferior corner of the superior vertebra and the inferior vertebra, the posteroinferior corner of the superior vertebra and the inferior vertebra and which was divided by 4 . For evaluation of disc endplates and disc degeneration, Modic changes and Pfirrmann grading were used, respectively (Tables 1, 2) $[1,5,11]$.
We analyzed and organized 2 patient groups according to the difference between initial and last measured disc height. Group A included patient with no or $<2 \mathrm{~mm}$ disc height (hereinafter referred to as not-changed disc height group). Group B included patients with $\geq 2 \mathrm{~mm}$ decrease in disc height (hereinafter referred to as decreased disc height group).

Lumbar MRI was performed twice spine position during this study with a 3.0 Tesla imager (Philips Medical Systems, Milwaukee, WI, USA) from T10 to S5. The MR protocol was adjusted by a radiologist. All images were uploaded to the in-hospital picture archive and communication system (PACS; Infinitt PACS, invent-

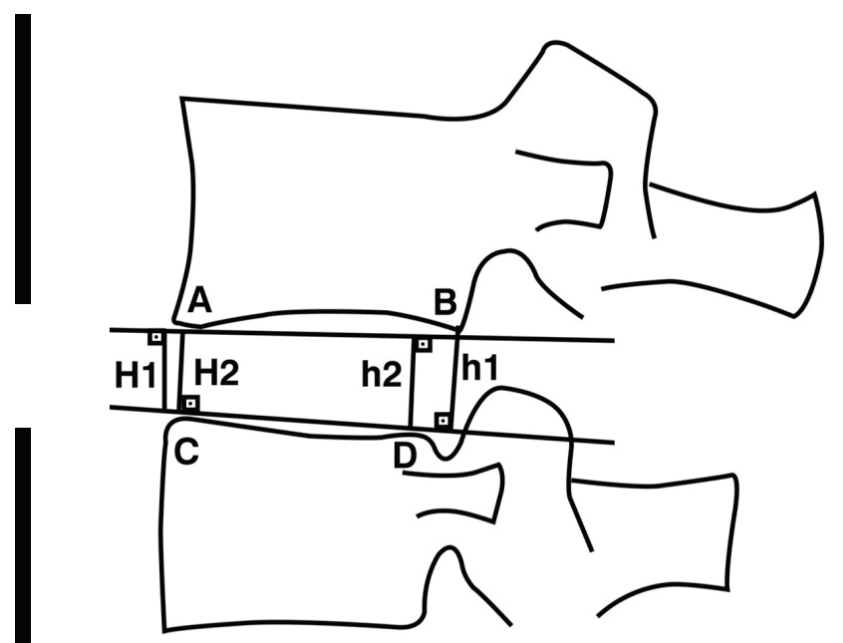

Fig. 1. Measurement of the intervertebral disc height using the modified Farfan method [4]. Disc height $=(\mathrm{H} 1+\mathrm{H} 2+\mathrm{h} 1+\mathrm{h} 2) / 4 . \mathrm{A}$, Anteroinferior corner of the superior vertebra; $B$, posteroinferior

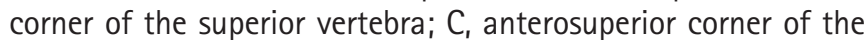
inferior vertebra; $D$, posterosuperior corner of the inferior vertebra.

Table 1. Endplate changes using the Modic classification

\begin{tabular}{lccc}
\hline Type & T1-weighted images & T2-weighted images & Description \\
\hline I & Low signal & High signal & Edema and inflammation of bone marrow \\
II & High signal & High signal & Marrow ischemia; yellow fatty marrow; transformation \\
III & Low signal & Low signal & Sclerosis over subchondral bony area \\
\hline
\end{tabular}

Table 2. Disc degeneration classification using the Pfirrmann grading system

\begin{tabular}{|c|c|c|c|c|}
\hline Grade & Structure & $\begin{array}{l}\text { Distinction of } \\
\text { nucleus and annulus }\end{array}$ & Signal intensity & Height of intervertebral disc \\
\hline 1 & Homogenous, bright white & Clear & $\begin{array}{l}\text { Hyperintense, isointense to cerebrospinal } \\
\text { fluid }\end{array}$ & Normal \\
\hline 2 & $\begin{array}{l}\text { Inhomogenous with or without horizontal } \\
\text { bands }\end{array}$ & Clear & $\begin{array}{l}\text { Hyperintense, isointense to cerebrospinal } \\
\text { fluid }\end{array}$ & Normal \\
\hline 3 & Inhomogenous, gray & Unclear & Intermediate & Normal to slightly decreased \\
\hline 4 & Inhomogenous, gray to black & Lost & Intermediate to hypointense & Normal to moderately decreased \\
\hline 5 & Inhomogenous, black & Lost & Hypointense & Collapsed disc space \\
\hline
\end{tabular}


ed by Infinitt Co., Seoul, Korea) and reviewed using the workstation-base unit (Hewlett-Packard Co., Palo Alto, CA, USA). All 112 MR images of 56 patients were reviewed independently by 2 experts ( 1 neurosurgeon and 1 radiologist) who had not been given any information about the subjects. Lumbar vertebrae were counted from S1. The status of disc herniation was defined as below: Protrusion of disc herniation is focal herniation which has a broader base than the body, while extrusion has a narrower base than the body. When there is already continuity loss with the disc itself, it is called disc sequestration [12].

\section{Analysis}

Clinical characteristics, including age, sex, smoking history and visual analogue scale (VAS, 10 points for back and leg) score were examined in each group. Radiologically, the level, status, laterality of lumbar disc herniation, Modic change, and disc degeneration grade were investigated between groups. Clinical and radiologic factors were compared to determine those that caused height differences between groups (Table 3). The patients group with decreased disc heights was analyzed about factor related to disc height decrease.

Table 3. Demographic and radiologic data of herniated lumbar disc patients

\begin{tabular}{|c|c|c|c|}
\hline Variable & Group A $(n=30)$ & Group B $(n=26)$ & P-value \\
\hline Sex (male:female) & 18:12 & 18:8 & \\
\hline Mean age (yr) & $68.2 \pm 11.3$ & $67.8 \pm 13.3$ & 0.072 \\
\hline \multicolumn{4}{|l|}{ Visual analogue scale (back pain) } \\
\hline Initial & 4.8 & 4.7 & 0.452 \\
\hline Follow-up & 2.4 & 2.3 & 0.448 \\
\hline \multicolumn{4}{|l|}{ Disc height (mm) } \\
\hline Initial & $12.15 \pm 0.15$ & $12.48 \pm 0.35$ & 0.132 \\
\hline Follow-up & $11.89 \pm 0.41$ & $10.23 \pm 0.82$ & $0.034^{*}$ \\
\hline Difference (initial-follow-up) & $0.88 \pm 0.75$ & $2.16 \pm 0.64$ & $0.021^{*}$ \\
\hline \multicolumn{4}{|l|}{ Modic change } \\
\hline Grade 3 & $1(3.3)$ & $1(3.8)$ & 0.082 \\
\hline \multicolumn{4}{|l|}{ Disc degeneration classification } \\
\hline Grade 1 & $14(46.7)$ & 0 & \\
\hline Grade 2 & $16(53.3)$ & 0 & \\
\hline Grade 3 & 0 & $8(30.8)$ & \\
\hline Grade 4 & 0 & $18(69.2)$ & \\
\hline Grade 5 & 0 & 0 & \\
\hline \multicolumn{4}{|l|}{ Level of disc herniation } \\
\hline $\mathrm{L} 3 / 4$ & $5(16.7)$ & $4(15.4)$ & 0.167 \\
\hline$\llcorner 4 / 5$ & $17(56.7)$ & $21(80.8)$ & 0.075 \\
\hline Left:right & $13: 17$ & $14: 12$ & \\
\hline
\end{tabular}

Values are presented as mean \pm standard deviation or number (\%).

Group A, patients with unchanged disc height; group B, patients with decreased disc height.

${ }^{a)}$ Visual analogue scale (10 points), ${ }^{b}$ months (interval between initial and follow-up magnetic resonance imaging).

${ }^{*} \mathrm{P}<0.05$. 
The clinical and radiologic analysis was performed using the IBM SPSS ver. 22.0 (IBM Corp., Armonk, NY, USA) using an independent samples t-test and chi-squared test. Statistical significance was measured to be when the P-value was less than 0.05 . The study was approved by the Ewha Womans University Mokdong Hospital Bioethics Committee (IRB No. 2020-06-16-0017).

\section{Results}

Patients ages ranged from 65 to 86 years (mean: $68.2 \pm 11.3$ years in group A, 67.8 \pm 13.3 in group B) (Table 3). The total group mean age was 75.2 years and the male to female ratio was 1.8:1 (36 males and 20 females). The initial VAS scores in group A and B were 4.8 and 4.7 points, respectively. The VAS scores at the time of last follow-up were 2.4 and 2.3 points in groups A and B. MRI scan interval was 13.2 and 14.3 months, respectively. There were no significant differences in age, sex ratio, smoking ratio, MRI scan interval, and VAS scores between groups.

The mean differences (initial disc height-last disc height) were $0.88 \pm 0.75 \mathrm{~mm}$ and $2.16 \pm 0.64 \mathrm{~mm}$ in group $\mathrm{A}$ and $\mathrm{B}$, respectively, which was significant $(P=0.021)$. However, patients were divided into 2 groups according to disc height difference (Table 3). Initial mean disc heights were $12.15 \pm 0.15 \mathrm{~mm}$ and $12.48 \pm 0.35 \mathrm{~mm}$ in group A and group B, respectively, which were not different between the 2 groups in disc height at the initial diagnosis. Last mean of disc heights was $11.89 \pm 0.41 \mathrm{~mm}$ in group $\mathrm{A}$ and $10.23 \pm 0.82$ $\mathrm{mm}$ in group $\mathrm{B}$. The initial disc height was no different between the 2 groups, but the last disc height of group B was less than group A (Table 3).

Level of disc herniation was observed in group A: 5 cases in $\mathrm{L} 3 / 4,17$ in $\mathrm{L} 4 / 5$, and 8 in $\mathrm{L5} / \mathrm{S} 1$ and in group B: 4 cases in L3/4, 21 in L4/5, and 7 in L5/S1. In both groups, disc herniation was the most common in L4/5, followed by L5/S1 and L3/4. Status of disc herniation revealed, there were 14 cases protrusion, 7 extrusions, and 9 sequestrations in group A. There were 5 cases protrusion, 10 extrusions, and 11 sequestrations in group $\mathrm{B}$. There were more protrusion cases (14/30) in group A than group B. In contrast, extrusion and sequestration was more common in group $\mathrm{B}$ (10/26 and $11 / 26$ cases, respectively. The ratio of laterality of disc herniation (left:right) were 13:17 and 14:12 in groups A and B, respectively. There were no significant statistical differences in level of disc herniation and laterality of disc herniation between groups (Table 3).

About Modic change, group A had more normal cases than group B: 24 cases (80.0\%) in not-changed disc height group, 13 cases $(50.0 \%)$ in decreased disc height group. According to disc degeneration classification, all cases of not-changed disc height group belonged to both grade 1 and grade 2 , and all cases of decreased disc height group belonged to grade 3, 4 and 5 .

\section{Discussion}

Lumbar disc herniation has been known to occur predominantly in L3/4, L4/5, and L5/S1 and can be further classified into protrusion, extrusion, and sequestration [13]. Disc protrusion was demarcated as a focal or asymmetric extension of the disc beyond the vertebral border, with the protruded origin wider than any other aspect of the protrusion. Extrusion was defined as a more extreme extension of the disc beyond the vertebral border, with the base against the disc of origin slenderer than the diameter of the extruding material and a connection between the material and the disc of origin. Sequestration was defined as a free disc fragment that was distinct from the origin disc [14].

In this study, we divided patients who were treated conservatively into 2 groups, according to their change in disc heights (Fig. 2).

It was observed that the decreased disc height patients group had more extrusion and sequestration cases than not-changed disc

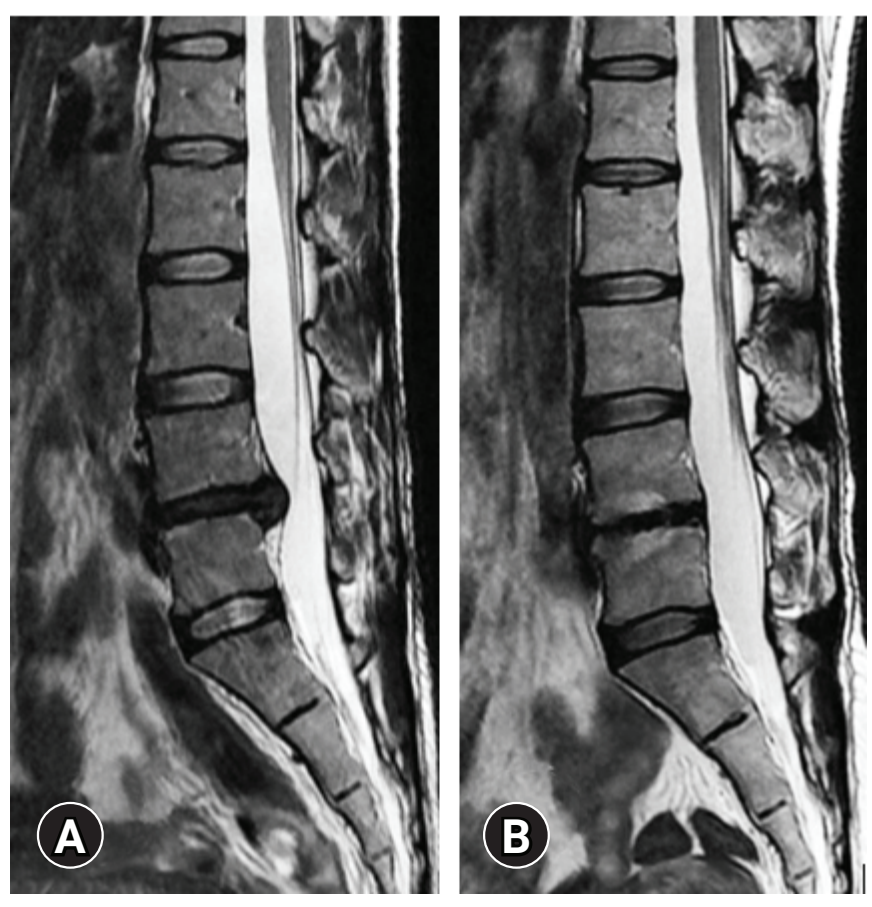

Fig. 2. A 32-year-old male with symptomatic lumbar disc hernation. He had suffered from mild back pain and left leg pain for 3 weeks. Magnetic resonance imaging (MRI) findings. (A) Initial $M R I$, showing a large area of disc extrusion at the $L 4 / 5$ disc space. (B) MRI taken 1 year after the initial MRI, showing spontaneous regression of the herniated lumbar disc and loss of disc height. Written informed consent was obtained for publication of this case report and accompanying images. 
height patients group (Table 3). These results suggested 2 things: first, by morphology of disc herniation, extrusion and sequestration are expected to have more herniated disc when compared with protrusion [12-14]. Secondly, extrusion and sequestration are likely to have a ruptured posterior longitudinal ligament (PLL) when compared with protrusion [10,12-14]. In general, it can be said that the higher the amount of herniation and the more the PLL supporting posterior aspect of disc ruptures, the higher the possibility that the disc height will be lowered later.

Several pathophysiological mechanisms have been reported for the natural regression of the herniated intervertebral disc; however, the exact mechanism has not been elucidated $[7,9]$. Three hypotheses have been proposed in the literature [5,9]. First, the prolapsed intervertebral disc returns to the intervertebral disc space by a healthy PLL. Second, the size of the intervertebral disc is reduced and resolved by gradual dehydration and contraction. Third, the herniated intervertebral disc causes immunologic, biochemical responses and neovascularization, which results in phagocytosis and enzymatic degradation of cartilage tissue. Among them, the most recently noted the hypothesis of spontaneous resolution is that intervertebral disc tissue is absorbed by immune responses and neovascularization, which be inferred from the rim enhancement of end plate of disc space on the Gadolinium enhanced MRI in patients with lumbar herniated disc [14].

In this study, decreased disc height patients group had more Modic change and disc degeneration than not-changed disc height patients group (Table 3 ). This suggested that more degenerative change cause disc height decrease later.

Surgical treatment is considered when there is persistent pain or neurological symptoms despite the proper period of conservative treatment [12]. Surgery creates a dramatic recovery of symptoms in lumbar intervertebral disc herniation; however, the incidence of recurrence or complications after surgery is reported to be $5 \%$ to $10 \%[8,14]$. Additionally, there have been reports that removal of a protruded lumbar intervertebral disc may accelerate degenerative changes in the intervertebral disc and end plates, resulting in a loss of disc space height, Modic change, and post-discectomy back pain $[13,15]$.

In this study, spontaneously resolved disc herniation without discectomy showed a decrease in disc height at long-term follow-up MRI. Conservative treatment is an effective treatment for herniated lumbar disc; however, it is necessary to recognize lumbar intervertebral disc herniation itself as a risk factor that can cause the loss of intervertebral disc height along with disc degeneration and Modic changes.

There were no differences in back pain between not-changed disc height patient and decreased disc height patient. To date, the relationship between disc height reduction or disc degeneration and back pain remains unclear $[11,16]$. In addition to discussing with patients about the options of conservative treatment for herniated lumbar disc, it is necessary to explain that herniated lumbar disc itself can cause a decrease of disc height and chronic back pain due to degenerative changes in the intervertebral disc.

Further research is required on the factors that are associated with degenerative changes in lumbar intervertebral disc herniation.

This study has some limitations. The main disadvantage is the retrospective nature. The measurements of disc height in supine position MRI do not reflect real disc height. We organized the patient groups and selected patients into groups, which creates a bias. Additionally, the number of patients in this study was small and the follow-up periods were relatively short to obtain significant conclusions. Therefore, further research with larger patient populations is required to fully elucidate the underlying mechanisms, and assess radiological and clinical evaluations.

\section{Conclusion}

In conservative treatment option for symptomatic lumbar disc herniation, decrease of disc height may occur later and it would be more at the cases of extrusion and sequestration-type herniation. This disc height decrease after lumbar disc herniation without surgical removal of disc may be due to disc degeneration and disc herniation itself. It is necessary to explain to patients who are treated conservatively that disc height may be reduced later.

\section{Conflicts of interest}

No potential conflict of interest relevant to this article was reported.

\section{ORCID}

Yongjae Cho, https://orcid.org/0000-0002-4498-1184

\section{REFERENCES}

1. Antar V, Baran O, Kelten B, et al. Morphometric analysis of lumbar disc space in the Turkish population and safe discectomy distance in lumbar disc surgery. Turk Neurosurg 2017;27:603-9.

2. Autio RA, Karppinen J, Niinimäki J, et al. Determinants of spontaneous resorption of intervertebral disc herniations. Spine (Phila Pa 1976) 2006;31:1247-52.

3. Froholdt A, Brox JI, Reikerås O, Leivseth G. Disc height and sagittal alignment in operated and non-operated levels in the lumbar 
spine at long-term follow-up: a case-control study. Open Orthop J 2013;7:258-63.

4. Guinto FC Jr, Hashim H, Stumer M. CT demonstration of disk regression after conservative therapy. AJNR Am J Neuroradiol 1984;5:632-3.

5. Kim DK, Oh CH, Lee MS, Yoon SH, Park HC, Park CO. Prevalence of lumbar disc herniation in adolescent males in Seoul, Korea: prevalence of adolescent LDH in Seoul, Korea. Korean J Spine 2011;8:261-6.

6. Komori H, Shinomiya K, Nakai O, Yamaura I, Takeda S, Furuya $\mathrm{K}$. The natural history of herniated nucleus pulposus with radiculopathy. Spine (Phila Pa 1976) 1996;21:225-9.

7. Kraemer J, Kolditz D, Gowin R. Water and electrolyte content of human intervertebral discs under variable load. Spine (Phila Pa 1976) $1985 ; 10: 69-71$.

8. McGirt MJ, Eustacchio S, Varga P, et al. A prospective cohort study of close interval computed tomography and magnetic resonance imaging after primary lumbar discectomy: factors associated with recurrent disc herniation and disc height loss. Spine (Phila Pa 1976) 2009;34:2044-51.

9. Neubert A, Fripp J, Engstrom C, Gal Y, Crozier S, Kingsley MI. Validity and reliability of computerized measurement of lumbar intervertebral disc height and volume from magnetic resonance images. Spine J 2014;14:2773-81.
10. Ohtori S, Yamashita M, Yamauchi K, et al. Low back pain after lumbar discectomy in patients showing endplate Modic type 1 change. Spine (Phila Pa 1976) 2010;35:E596-600.

11. Teichtahl AJ, Urquhart DM, Wang Y, et al. Modic changes in the lumbar spine and their association with body composition, fat distribution and intervertebral disc height: a 3.0 T-MRI study. BMC Musculoskelet Disord 2016;17:92.

12. Postacchini F. Lumbar disc herniation: a new equilibrium is needed between nonoperative and operative treatment. Spine (Phila Pa 1976) 2001;26:601.

13. Roberts N, Gratin C, Whitehouse GH. MRI analysis of lumbar intervertebral disc height in young and older populations. J Magn Reson Imaging 1997;7:880-6.

14. Shahmohammadi MR, Behrouzian S. Effect of preoperative Modic change in the outcome of patients with low back pain following posterior spinal fusion or laminectomy. Asian J Neurosurg 2019;14:432-5.

15. Slavin KV, Raja A, Thornton J, Wagner FC Jr. Spontaneous regression of a large lumbar disc herniation: report of an illustrative case. Surg Neurol 2001;56:333-7.

16. Turk O, Antar V, Yaldiz C. Spontaneous regression of herniated nucleus pulposus: the clinical findings of 76 patients. Medicine (Baltimore) 2019;98:e14667. 